\title{
Incidence of Medial Meniscal Tears in Various Age Groups
}

\author{
Ceylan Çolak' (D), Subhas Naveen ${ }^{1}$ (1), Jennifer Bullen² (D), Hakan Ilaslan (1) \\ ${ }^{1}$ Department of Radiology, Cleveland Clinic, Cleveland, USA \\ ${ }^{2}$ Cleveland Clinic, Quantitative Health Sciences, Cleveland, USA
}

Cite this article as: Çolak C, Naveen S, Bullen J, ilaslan H. Incidence of Medial Meniscal Tears in Various Age Groups. JAREM 2018; 8(3): 203-6.

\begin{abstract}
Objective: Meniscal tears are very common and are a major risk factor for knee osteoarthritis development. The aim of this study was to ascertain the incidence of medial meniscal tears in magnetic resonance imaging (MRI) studies and to determine whether increasing age affects the likelihood of a patient having a meniscal tear.

Methods: All patients who underwent knee MRI at our institution between January 3, 2017, and December 30, 2017, were included in the study. Radiological reports of magnetic resonance images were assessed for evidence of any kind of medial meniscus tear. A logistic regression model was used to assess the effect of age and sex on the probability of a patient having a medial meniscal tear.

Results: A total of 1855 patients with a medial meniscal tear and 1935 patients without a medial meniscal tear were included in this study. The logistic regression model showed that the probability of having a medial meniscal tear increased with age in both men and women. In this sample, 52\% (923/1767) of men had a medial meniscal tear versus $46 \%(932 / 2024)$ of women; men were more likely to have a medial meniscal tear than women (odds ratio, $1.28 ; 95 \% \mathrm{Cl}, 1.13-1.46 ; \mathrm{p}<0.001$ ).

Conclusion: The incidence of medial meniscal tears increases with age. Therefore, radiologists and orthopedic surgeons interpreting MRI studies should remember that the existence of a medial meniscal tear in an elderly patient may be an incidental finding and may not explain the patient's symptoms.
\end{abstract}

Keywords: Meniscus, medial meniscus tear, knee magnetic resonance imaging (MRI)

ORCID IDs of the authors: C.C. 0000-0002-8278-9302; S.N. 0000-0002-0502-6280; B.J. 0000-0002-5403-2935; i.H. 0000-0002-8152-3625.

\section{INTRODUCTION}

The menisci are intra-articular structures that have complex biomechanic functions, such as shock absorption and load distribution $(1,2)$. Meniscal pathologies, especially symptomatic and asymptomatic meniscal tears, are the most prevalent intra-articular knee injury in the United States and are also the most common indication for knee surgery (3-5). Meniscal tear in particular is one of the important risk factors for the development and progression of knee osteoarthritis, regardless of tear type (6-8). Conversely, osteoarthritis can lead to meniscal tear in some patients (1). Research has shown that meniscal tears occur more frequently in the medial meniscus than in the lateral meniscus (9).

Magnetic resonance imaging (MRI) is often used to diagnose meniscal tears. Clinicians also use MRI findings to determine whether a patient should undergo arthroscopic meniscectomy. Several previous studies have used MRI findings to assess the potential relationships between patient age and sex, and the incidence of meniscal tears (10-14). However, there is little information available regarding the incidence of medial meniscal tears specifically and the effect of patient age and sex on the incidence of these tears. In this study, we assessed knee MRI reports to ascertain the overall incidence of medial meniscal tears and to determine whether increasing age affects the likelihood of a patient having a meniscal tear.

\section{METHODS}

For this retrospective study, we used REDCap (a secure web application for building and managing online surveys and databases) to conduct a search of the Musculoskeletal Radiology Research Laboratory database for relevant patient records. This study was exempt from Institutional Review Board (IRB) approval because of using the existing records on REDCap. All patients who underwent knee MRI for any indication at our institution, between January 3, 2017, and December 30, 2017, were considered for inclusion in this study. All radiological reports of MR scans were assessed for evidence of any kind of medial meniscal tear regardless of whether the patient had any other knee pathologies.

\section{Statistical Analysis}

A logistic regression model was used to assess the effect of age and sex on the probability of a patient having a medial meniscal 


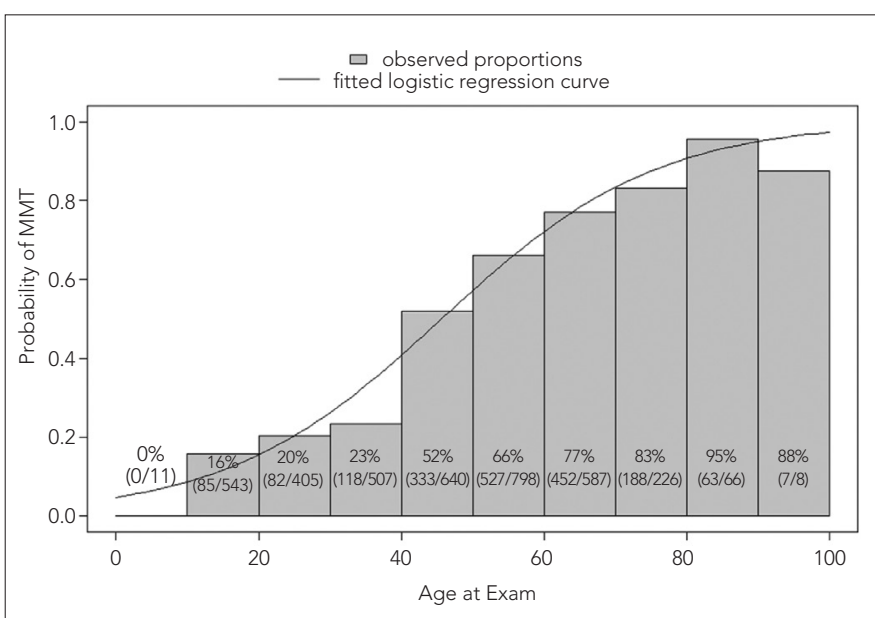

Figure 1. The probability of having a medial meniscal tear increases with age. Observed proportions from this sample are shown as gray bars. The fitted logistic regression curve is also shown

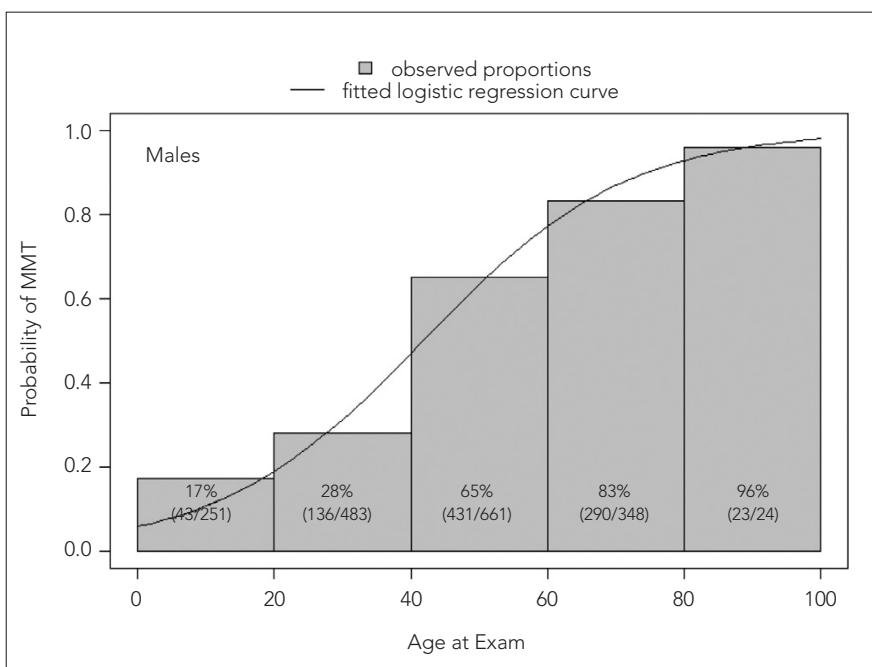

Figure 3. The probability of having a medial meniscal tear increases with age in men. Observed proportions from this sample are shown as gray bars. The fitted logistic regression curve is also shown

tear. Age, sex, and the interaction between age and sex were all included as predictors in the model. The interaction between age and sex was then dropped from the model, as this factor was not significant at the 0.10 level. The results presented are therefore based on a model with two main effects: age and sex. Only cases with complete records were included; one patient with unknown sex was excluded from the analysis.

\section{RESULTS}

A total of 3790 patients (1767 men; 2023 women) were included in the study. From the study patients, 1855 had a medial meniscal tear, and 1935 did not have a medial meniscal tear. The overall age of the patients was divided into 10- and 20-year interval subgroups (Figure 1). The most patients were in the $40-60$ year age subgroup

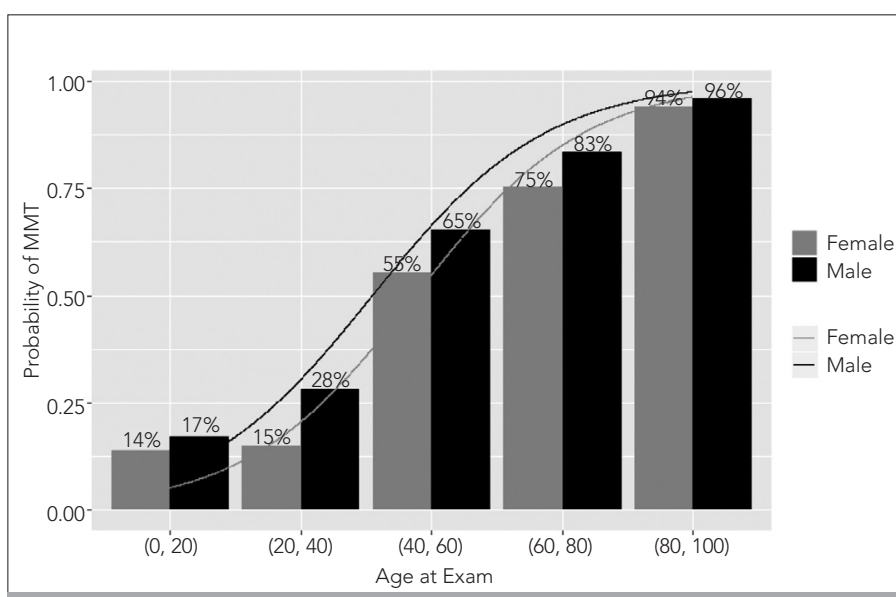

Figure 2. The probability of having a medial meniscal tear in men and women. Observed proportions from this sample are shown as orange (women) and green (men) bars. The fitted logistic regression curve is also shown using the same colors

As age increased, the odds of having a medial meniscal tear increased (Figure 1). According to the model, the odds of having a medial meniscal tear nearly doubled with each 10-year increase in age (odds ratio, 1.94; 95\% confidence interval (Cl), 1.85-2.03; $\mathrm{p}<0.001$ ). Additionally, men were more likely to have a medial meniscal tear than women (odds ratio, 1.28; $95 \% \mathrm{Cl}, 1.13-1.46$; $\mathrm{p}<0.001)$ (Figure 2). In this sample, 52\% (923/1767) of men had a medial meniscal tear (Figure 3) versus 46\% (932/2024) of women (Figure 4).

\section{DISCUSSION}

With this model, we found that the probability of a medial meniscal tear occurring increases with increasing age regardless of patient sex, although men were more likely to have a medial meniscal tear than women.

In a previous study of randomly selected adults assessed without regard for symptoms, Englund et al. (10) similarly presented that the prevalence of meniscal tears increases with increasing age and is higher in men. Mansori et al. (12) also reported a higher risk for meniscal tears among older men (12). Meniscal tears in elderly patients likely result from long-term degeneration $(10,11)$; the higher incidence of tears among men may be related to an increased risk of injuries from playing male-dominated contact sports or working in fields that require strenuous physical activity. Interestingly, Hwang et al. (13) found that women are more likely to have medial meniscus root tears. In this study, we did not differentiate among types of medial meniscal tears.

Most meniscal tears are asymptomatic (1). Therefore, it is important to determine whether knee symptoms are caused by a meniscal tear or another pathology, especially in elderly patients. Meniscal tears are extremely rare in young children; in this study, no medial meniscal tears were seen among patients in the first decade of life. On the other hand, lateral meniscus tears are more frequent than medial meniscal tears in younger patients and are likely to be associated with trauma (14). 


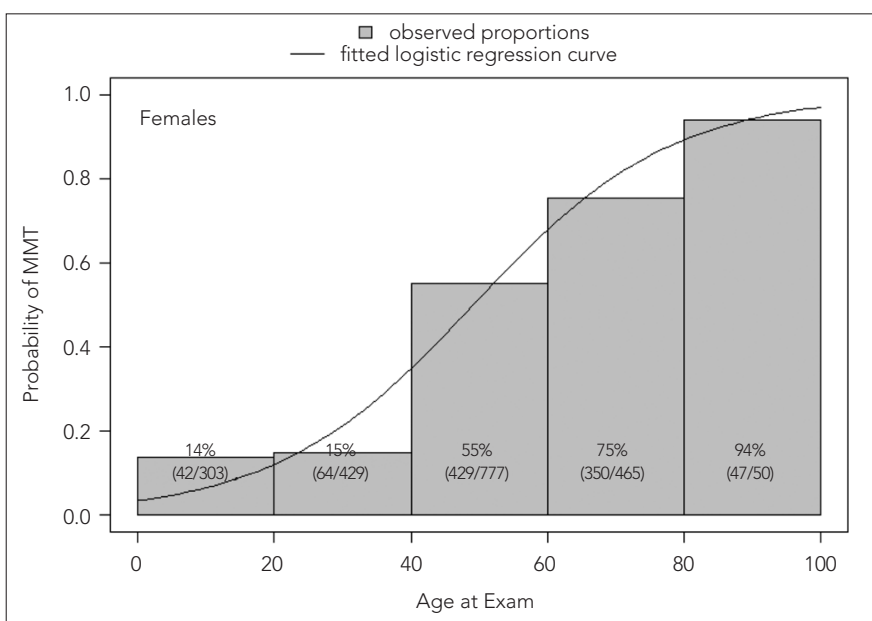

Figure 4. The probability of having a medial meniscal tear increases with age in women. Observed proportions from this sample are shown as gray bars. The fitted logistic regression curve is also shown.

Magnetic resorance imaging is a useful imaging modality for the evaluation of meniscal tears, demonstrating a sensitivity of $93 \%$ and a specificity of $88 \%$ to diagnose medial meniscal tears (15). With the model described in this study, we were able to use knee MRI results to show the relationship between the medial meniscal tear and age/sex. Nearly every patient in the study older than 80 years demonstrated evidence of a medial meniscal tear on knee MRI. Because medial meniscal tears are so common among these patients, evidence of a tear on knee MRI may be an incidental finding and may not explain the patient's symptoms. Radiologists and orthopedic surgeons should therefore remember that elderly patients who present with knee pain should not undergo expensive MRI studies based solely on a suspicion of meniscal tear, as most of these patients will have a medial meniscal tear regardless of the presence or absence of symptoms. These patients should be examined carefully for the presence of the other pathologies related to the knee pain.

Our model was limited by including only patients who underwent knee MRI regardless of the presence of any meniscal tear symptoms; this may have affected our calculations regarding the real incidence of medial meniscal tears. Additionally, this was a retrospective review of scans that were performed on various 1.5 and 3 Tesla MRI systems and reviewed by various radiologists. These variations may also have affected our calculations.

\section{CONCLUSION}

We demonstrated that the incidence of medial meniscal tears increases with age. Nearly all patients older than 80 years demonstrated evidence of a medial meniscal tear on knee MRI; therefore, MRI findings of a medial meniscal tear in elderly patients may be incidental and may not explain the patient's symptoms.

Ethics Committee Approval: This study was exempt from the Institutional Review Board approval because of using the existing records on REDCap.
Informed Consent: Informed consent was not taken from patients due to the retrospective nature of the study.

Peer-review: Externally peer-reviewed.

Author Contributions: Concept - H.I., N.S.; Design - H.I., N.S.; Supervision - H.I., N.S.; Resources - J.B., N.S.; Materials - N.S., J.B., H.I.; Data Collection and/or Processing - C.C., H.I., J.B.; Analysis and/or Interpretation - C.C., H.I.; Literature Search - C.C.; Writing Manuscript - C.C.; Critical Review - C.C., H.i.

Acknowledgements: The authors would like to thank Megan Griffiths.

Conflict of Interest: The authors have no conflict of interest to declare.

Financial Disclosure: The authors declared that this study has received no financial support.

\section{REFERENCES}

1. Englund M, Roemer FW, Hayashi D, Crema MD, Guermazi A. Meniscus pathology, osteoarthritis and the treatment controversy. Nat Rev Rheumatol 2012; 8: 412-9. [CrossRef]

2. Walker PS, Erkman MJ. The role of the menisci in force transmission across the knee. Clin Orthop Relat Res 1975; 109: 184-92. [CrossRef]

3. Bhattacharyya T, Gale D, Dewire P, Totterman S, Gale ME, McLaughlin $S$, et al. The clinical importance of meniscal tears demonstrated by magnetic resonance imaging in osteoarthritis of the knee. J Bone Joint Surg Am 2003; 85-A: 4-9. [CrossRef]

4. Morgan CD, Wojtys EM, Casscells CD, Casscells SW. Arthroscopic meniscal repair evaluated by second-look arthroscopy. Am J Sports Med 1991; 19: 632-7. [CrossRef]

5. Salata MJ, Gibbs AE, Sekiya JK. A systematic review of clinical outcomes in patients undergoing meniscectomy. Am J Sports Med 2010; 38: 1907-16. [CrossRef]

6. Englund M, Guermazi A, Lohmander SL. The role of the meniscus in knee osteoarthritis: a cause or consequence? Radiol Clin North Am 2009; 47: 703-12. [CrossRef]

7. Englund M. Meniscal tear-a feature of osteoarthritis. Acta Orthop Scand Suppl 2004; 75: 1-45. [CrossRef]

8. Englund M, Guermazi A, Roemer FW, Aliabadi P, Yang M, Lewis $C E$, et al. Meniscal tear in knees without surgery and the development of radiographic osteoarthritis among middle-aged and elderly persons: the Multicenter Osteoarthritis Study. Arthritis Rheum 2009; 60: 831-9. [CrossRef]

9. Lewandrowski KU, Müller J, Schollmeier G. Concomitant meniscal and articular cartilage lesions in the femorotibial joint. Am J Sports Med 1997; 25: 486-94. [CrossRef]

10. Englund M, Guermazi A, Gale D, Hunter DJ, Aliabadi P, Clancy M, et al. Incidental meniscal findings on knee MRI in middle-aged and elderly persons. N Engl J Med 2008; 359: 1108-15. [CrossRef]

11. Barrett GR, Field MH, Treacy SH, Ruff CG. Clinical results of meniscus repair in patients 40 years and older. Arthroscopy 1998; 14: 824-9. [CrossRef]

12. Mansori AE, Lording T, Schneider A, Dumas R, Servien E, Lustig S. Incidence and patterns of meniscal tears accompanying the anterior cruciate ligament injury: possible local and generalized risk factors. Int Orthop 2018; 42: 2113-21. [CrossRef]

13. Hwang BY, Kim SJ, Lee SW, Lee HE, Lee CK, Hunter DJ, et al. Risk factors for medial meniscus posterior root tear. Am J Sports Med 2012; 40: 1606-10. [CrossRef] 
14. Yeh PC, Starkey C, Lombardo S, Vitti G, Kharrazi FD. Epidemiology of isolated meniscal injury and its effect on performance in athletes from the National Basketball Association. Am J Sports Med 2012; 40: 589-94. [CrossRef]
15. Blake MH, Lattermann C, Johnson DL. MRI and Arthroscopic Evaluation of Meniscal Injuries. Sports Med Arthrosc Rev 2017; 25: 219-26.

[CrossRef] 